1. Myolinakis E, Hohmann EL, Calderwood SB. Central nervous system infection with Listeria monocytogenes. 33 years' experience at a general hospital and review of 776 episodes from the leterature. Medicine 1998; 77: 313-336.

2. Alcoba Leza M, Carro Fernández JA, Pérez Simón MR, Guerra Laso J, Alonso Fernández A, Arto Millán L. Meningitis por Listeria monocytogenes en el adulto en España. Presentación de 10 casos y revisión de la literatura. Rev Clin Esp 2002; 202: 638-643.

3. Seidel Padilla V, Martínez-Roig A, Esteban Torne E, Herrero Pérez S, López Segura N, Santos Solis A, Bonet Alcaina M. Meningitis por Listeria monocytogenes en una niña inmunocompetente. An Pediatr (Barc) 2004; 60: 184-185.

4. Staudinger R, Levine D, Swaminathan B, Zagzag D. Neurolisteriosis presenting as recurrent transient ischemic attacks. Ann Neurol 2000; 48: 661-665.

5. Gerl A, Mittermuller J, Bise K, Wilmanns W. Listeriosis in malignant diseases. Dtsch Med Wochenschr 1991; 116: 1144-1148.

6. Hardin SG, Erwin PC, Patterson L, New D, Graber C, Halford SK. Clinical comparisons of La Crosse encephalitis and enteroviral central nervous system infections in a pediatric population: 2001 surveillance in East Tennessee. Am J Infect Control 2003; 31: 508-510.

7. López Cortés LF, Cruz Ruiz M, Gómez Mateos J, Jiménez Hernández D, Jiménez Mejias E, Pachón J, et al. Adenosine deaminase activity in the CSF of patients with aseptic meningitis: utility in the diagnosis of tuberculous meningitis or neurobrucellosis. Clin Infect Dis 1995; 20: 525-530.

8. Choi SH, Kim YS, Bae IG, Chung JW, Lee MS, Kang JM, et al. The possible role of cerebrospinal fluid adenosine deaminase activity in the diagnosis of tuberculous meningitis in adults. Clin Neurol Neurosurg 2002; 104: 10-15.

9. Corral I, Quereda C, Navas E, Martín Dávila P, Pérez Elias MJ, Casado JL, et al. Adenosine deaminase activity in cerebrospinal fluid of HIVinfected patients: limited value for diagnosis of tuberculous meningitis. Eur J Microbiol Infect Dis 2004; 23: 471-476.

10. Jurado RL, Farley MM, Pereira E, Harvey RC, Schuchant A, Wenger JD, Stephens DS. Increased risk of meningitis and bacteriemia due to Listeria Monocytogenes in patients with Human Inmunodeficiency virus infection.Clin Infect Dis 1993; 17: 224-227.

\section{Pileflebitis idiopática en paciente con mutación del factor V Leiden}

\section{Sr. Director:}

La pileflebitis o tromboflebitis séptica de la vena porta es una complicación poco frecuente, aunque muy grave, de las infecciones intraabdominales que puede preceder al desarrollo de abscesos hepáticos. La causa más frecuente es la diverticulitis, seguida de la apendicitis y la enfermedad inflamatoria intestinal (1-4). La vena mesentérica superior se afecta sólo en el 34\% de los casos y puede ocasionar isquemia intestinal, infarto o muerte (4). La forma idiopática es rara, representa un tercio de los casos, y se presenta como un cuadro de dolor abdominal inespecífico $(5,6)$. Presentamos un caso de pileflebitis idiopática con afectación de la vena mesentérica superior en una paciente portadora de una mutación del factor V Leiden.

Mujer de 28 años de edad con antecedentes de parto natural hace diez meses, fumadora de 20 cigarrillos/día y a tratamiento con anticonceptivos orales ( $3 \mathrm{mg}$ de drospirenona/ $30 \mu \mathrm{grs}$ de etinilestradriol) que consulta por un cuadro de tres semanas de evolución de dolor abdominal, náuseas, vómitos, escalofríos y fiebre termometrada de hasta $40^{\circ} \mathrm{C}$, que cede con antitérmicos.
En la exploración física presentaba: $\mathrm{T}^{\mathrm{a}} 38,7{ }^{\circ} \mathrm{C}$, PA125/80 mmHg, P109 lpm y un abdomen doloroso a la palpación en epigastrio y región periumbilical izquierda, sin signos de irritación peritoneal, siendo el resto de la exploración por aparatos anodina. En las pruebas complementarias destacaban 15050 leucocitos ( $85 \%$ neutrófilos), bilirrubina $0,9 \mathrm{mg} / \mathrm{dl}$, GOT $16 \mathrm{U} / \mathrm{L}$, GPT 21 U/L, GGT $290 \mathrm{U} / \mathrm{L}$, fosfatasa alcalina $210 \mathrm{U} / \mathrm{L}$, protrombina $66 \%$, fibrinógeno $754 \mathrm{mg} / \mathrm{dL}$ y VSG de $91 \mathrm{~mm}$ en la $1^{\mathrm{a}}$ hora. La analítica elemental de orina, la radiografía de tórax y abdomen eran normales y los hemocultivos negativos. La ecografía y el TAC abdominal demostraron la existencia de trombosis de la vena mesentérica superior hasta su confluencia con la vena esplénica, trombosis de la vena porta principal y de su rama izquierda. La enteroclisis y la colonoscopia resultaron normales. El estudio de la coagulación objetivó la presencia de mutación heterocigota del factor V Leiden (1691G/1691A) y resistencia a la proteína C activada. Se realizó tratamiento antibiótico con piperacilina-tazobactam, durante 4 semanas, y tratamiento anticoagulante, inicialmente con heparina sódica y posteriormente con acenocumarol.

La pileflebitis es una complicación poco frecuente de las infecciones intraabdominales con una mortalidad del 11 al $32 \%$ $(1,4,7)$. La causa más frecuente es la diverticulitis, seguida de la apendicitis y la enfermedad inflamatoria intestinal (1-4). La forma idiopática representa un tercio de los casos (1) y se asocia a alteraciones de los factores de la coagulación, enfermedades malignas o sida (4-7). El agente etiológico más frecuente es el Bacteroides spp., sobre todo B. fragilis, seguido por los bacilos gramnegativos y estreptococos aerobios. Los hemocultivos son positivos en el 23 al $88 \%$ de los casos $(1,4)$. Debe sospecharse ante un cuadro de dolor abdominal, fiebre y escalofríos que se acompañe de leucocitosis, aunque pueden ser normales o incluso presentar leucopenia, alteración de la coagulación y elevación de las enzimas hepáticas, especialmente de la fosfatasa alcalina de tres a cuatro veces su valor y de la GGT de cinco a diez veces (1$4,6,8)$. La ictericia es infrecuente a menos que se asocie a colangitis o abscesos hepáticos (1). El diagnóstico precisa la demostración de trombosis de la vena porta en un paciente febril. La ecografía abdominal suele mostrar la presencia de material ecogénico en el interior de la vena porta y puede utilizarse para evaluar la progresión o detectar la recanalización. El TAC se ha utilizado para confirmar el diagnostico de sospecha y es útil para descartar la presencia de focos infecciosos intraabdominales así como para detectar la presencia de abscesos hepáticos $(2,3,6,8)$.

El tratamiento antibiótico debe ser efectivo frente a bacilos gramnegativos y anaerobios, especialmente B. fragilis, y administrarse durante 4 a 6 semanas $(1,2,4,6)$. Se han descrito casos de colocación de un catéter en la vena porta para el drenaje de la tromboflebitis supurada con buenos resultados (5). En caso de existir abscesos hepáticos la asociación de antibióticos y drenaje percutáneo parece el tratamiento más indicado, con una tasa de supervivencia global del 90\% (9). Aunque aún no existe consenso sobre el tratamiento anticoagulante, la mayoría de los autores lo recomiendan en caso de que exista un estado de hipercoagulabilidad, afectación de la vena mesentérica superior, extensión de la trombosis o fiebre persistente que no responde al tratamiento antibiótico $(2,4,8,10)$. En el caso que presentamos se realizó tratamiento anticoagulante, inicialmente con heparina sódica y posteriormente con acenocumarol, presentando en el momento del alta recanalización de la rama izquierda de la vena porta y de la vena mesentérica superior.

R. López Rodríguez, C. Martínez Rey, J. Campos Franco, M. R. Alende Sixto, J. A. Torre Carballada

Servicio de Medicina Interna. Complejo Hospitalario Universitario de Santiago de Compostela. Santiago de Compostela. A Coruña 
1. Plemmons RM, Dooley DP, Longfield RN. Septic thrombophlebitis of the portal vein (pylephlebitis): Diagnosis and management in the modern era. Clin Infect Dis 1995; 21: 1114-20.

2. Singh P, Yadav N, Visvalingam V, Indaram A, Bank S. Pylephlebitis: Diagnosis and management. Am J Gastroenterol 2001; 96: 1312-3.

3. Saxena R, Adolph M, Ziegler JR, et al. Pylephlebitis: a case report and review of outcome in the antibiotic era. Am J Gastroenterol 1996; 91: 1251-6.

4. Baril N, Wren S, Radin R, Ralls P, Stain S. The role of anticoagulation in pylefphlebitis. Am J Surg 1996; 172: 449-53.

5. Ohtake H, Urayama H, Nozaki Z, Harada T, Kawasuji M, Wantanabe Y. Surgical drainage for idiopathic suppurative pylephlebitis. Ann Vasc Surg 1998; 12: 83-5.

6. Sillero JM, Calvet X, Musulen E, Diaz-Ruiz MJ, Tolosa C, Pardo A Corcuera A, Malet A, Font J. Idiopathic pylephlebitis and idiopathic sclerosing peritonitis in a man with protein $\mathrm{S}$ deficiency. J Clin Gastroenterol 2001; 32: 262-5.

7. Etienne M, Gueit I, Abboud P, Pons JL, Jacquot S, Caron F. Fusobacterium nucleatum hepatic abscess with pylephlebitis associated with idiopathic CD4+ T lymphocytopenia. Clin Infect Dis 2001; 32: 326-8.

8. Duffy FJ, Millan MT, Schoetz DJ, Larsen CR. Suppurative pylephlebitis and pylethrombosis: the role of anticoagulation. Am Surgeon 1995; 61: 1041-4.

9. Seeto RK, Rockey DC. Pyogenic liver abscess. Changes in etiology, management and outcome. Medicine 1996; 75: 99-106.

10. Condat B, Pessione F, Denninger MH, Hillaire S, Valla D. Recent portal and mesenteric venous thrombosis: increased recognition and frecuent recanalization on anticoagulant therapy. Hepatology 2000; 32: 466-70.

\section{Infarto mesentérico de etiología no oclusiva}

\section{Señor Director:}

La isquemia mesentérica no oclusiva es un tipo de isquemia mesentérica poco frecuente que presenta unas características diferenciales, y a menudo su diagnóstico se realiza por exclusión (1). Presentamos nuestra experiencia en esta patología.

Paciente de 82 años intervenido de carcinoma de laringe 4 días previos a su ingreso en UCI, con buena evolución en el postoperatorio inmediato. Presenta súbitamente en planta emisión de vómitos de considerable cuantía $(1.500 \mathrm{cc})$, así como crisis de arritmia completa por fibrilación auricular a 200 por minuto, con gran descenso en las cifras de tensión arterial $(50 / 30 \mathrm{mmHg})$, por lo que ingresa en la Unidad de Cuidados Intensivos, donde además de lo mencionado se aprecia dolor abdominal difuso de manera espontánea y también a la palpación. Se implanta sonda nasogástrica obteniendo un elevado débito por la misma (2.000 cc en las primeras 24 horas). Se instaura tratamiento con expansores del plasma (coloides) y posteriormente con drogas vasoactivas ante la refractariedad para la corrección de la tensión arterial. Así mismo se asocia amiodarona en perfusión continua para disminuir la frecuencia cardíaca. Ante desaturación importante es preciso iniciar ventilación mecánica. Se recibe analítica con valores incrementados de las enzimas hepáticas (AST 300 U/L; ALT $267 \mathrm{U} / \mathrm{L})$, urea $(1,30 \mathrm{~g} / \mathrm{l})$ y creatinina $(4 \mathrm{mg} / \mathrm{dl})$, encontrando también alteraciones en la coagulación (tiempo de protrombina 16 seg. actividad de protrombina $62,4 \%$, INR 1,49 , tiempo de tromboplastina parcial activada $36 \mathrm{seg}$, fibrinógeno $161 \mathrm{mg} / \mathrm{dl}$, plaquetas $55 \mathrm{mill} / \mathrm{mm}^{3}$ ). Se realiza una TAC abdominal de urgencia donde se aprecia una distensión generalizada de las asas intestinales, sin patología obstructiva subyacente, hallando unos infartos isquémicos múltiples en el parénquima hepático y esplénico
(Fig. 1). No existen alteraciones en la raíz de las ramas de la aorta. Como la situación persiste tras 24 horas de su ingreso, se realiza una laparotomía exploradora en la que no se objetiva sufrimiento de las asas intestinales ni anomalías en la vasculatura abdominal. El paciente evoluciona satisfactoriamente pudiendo retirar drogas vasoactivas y apoyo respiratorio tras una semana de evolución, con corrección de todas las alteraciones analíticas mencionadas.

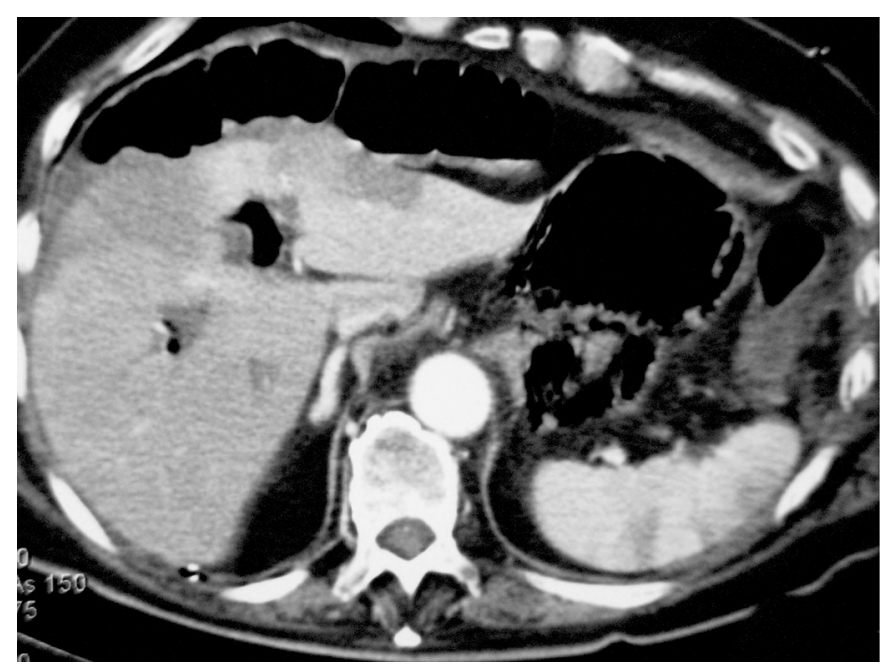

Fig. 1.TAC abdominal: distensión de asas intestinales. Infarto isquémico en parénquima hepático.

La isquemia mesentérica no oclusiva afecta a pacientes con edad superior a 50 años, constituye el $20-30 \%$ de todos los casos de isquemia mesentérica y posee una mortalidad del $50 \%$ que se incrementa con la edad, llegando a ser del $100 \%$ en los pacientes octogenarios $(1,2)$.

Habitualmente en su desarrollo concurren factores como insuficiencia cardiaca congestiva, insuficiencia aórtica, o fibrilación auricular, siendo característica la existencia de una intervención quirúrgica reciente (1). Puede existir, aunque no siempre, dolor abdominal como forma de presentación clínica, así como la presencia de íleo intestinal (ambos existentes en el caso presentado) (1,2).

$\mathrm{Su}$ mecanismo etiopatogénico reside en un fallo cardiaco agudo que origina una hipoperfusión intestinal a la que contribuyen significativamente la existencia de una fibrilación auricular o de una cirugía reciente, ambos presentes en nuestro caso. La fibrilación auricular en este tipo de isquemia, no juega un papel embolígeno, sino que es la responsable directa de una disminución en el flujo sanguíneo de la arteria mesentérica, lo que provoca una hipoperfusión de dicho territorio secundaria a la reducción de la función ventricular izquierda y por tanto de la fracción de eyección $(1,3)$. Por este motivo, es típica la aparición de isquemia en otros órganos abdominales, coexistiendo sincrónicamente infartos a nivel hepático, esplénico o renal (lo que explicaría los múltiples infartos hepáticos y esplénicos que se hallaron en el paciente) (1). La cirugía reciente es otro factor de riesgo para desarrollar este tipo de isquemia, probablemente por que comporta una pérdida de fluidos a menudo desapercibida, y un desarrollo de hipotensión arterial, ambos más frecuentes en pacientes ancianos (1). En ocasiones, existe también un vasoespasmo asociado de las arterias implicadas que se origina como mecanismo compensador, y que no se resuelve hasta que no se realiza la corrección de los factores precipitantes que han desencadenado la isquemia; dicho vasoespasmo puede también 